\title{
On localization effects in underdoped cuprates
}

\author{
C. Castellani, P. Schwab, and M. Grilli \\ Istituto di Fisica della Materia e Dipartimento di Fisica, Università "La Sapienza", piazzale A. \\ Moro 2, 00185 Roma - Italy
}

(May 13, 2018)

\begin{abstract}
We comment on transport experiments in underdoped $\mathrm{LaSrCuO}$ in the non-superconducting phase. The temperature dependence of the resistance strongly resembles what is expected from standard localization theory. However this theory fails, when comparing with experiments in more detail.
\end{abstract}

\section{INTRODUCTION}

It is generally believed that the understanding of the anomalous normal state properties would shed light on the understanding of the pairing mechanism of high- $T_{c}$ superconductors. In particular, low temperature transport experiments in the normal state should provide valuable informations on the physical mechanisms acting in high- $T_{c}$ materials. The normal state is usually inaccessible at low temperature, due to the onset of superconductivity. However, superconductivity can be suppressed down to lowest temperatures by applying a sufficiently high magnetic field.

In this paper we shall discuss the low temperature resistance in underdoped $\mathrm{La}_{2-\mathrm{x}} \mathrm{Sr}_{\mathrm{x}} \mathrm{CuO}_{4}$ (LSCO) at high magnetic fields, where insulating behavior with a typical $\log T$ dependence of the resistance has been observed [1]. Various proposals have been made for the origin of this behavior. Anderson et al. 22 interpreted the data in the framework of Luttinger liquid transport theory, which predicts a power law in the c-axis and in the in-plane conductivity $\sigma_{\mathrm{c}} \propto \sigma_{\mathrm{ab}} \propto T^{2 \alpha}$. Although it is not unreasonable to fit the experiments by a power law, much better fits are achieved assuming the logarithmic behavior. Alexandrov [3] reported a logarithmically divergent resistivity in a bipolaron model in presence of disorder, however this model has been repeatedly questioned [4]. Varma [5] argued that in a non Fermi-liquid even small disorder drives the density of states to zero and thus drives the system to an insulator.

Standard localization effects have been discussed as source of the increasing resistance since the first experiments [6, 1]. Evidence against this interpretation has arised from measurements of the Hall resistance [7], which is nearly temperature independent. However, since the mechanism which dominates the Hall effect in the cuprates is not clear, it is hard to make conclusions. So far a detailed analysis of the temperature dependence of the resistivity versus the predictions of localization theory has not been given. This will be subject of the present paper. 
In the next section we shall briefly recall standard localization theory in two dimensional systems, discussing both coherent backscattering and interaction effects. Then we shall apply localization theory to LSCO. We will demonstrate that the cross-over from 3d to $2 \mathrm{~d}$ localization in LSCO is expected near optimal doping. However the amplitude of the increase of resistance in that material in the underdoped region does not fully agree with standard 2d localization theory. We will argue that anomalous localization effects are indeed to be expected in that region in the presence of a disordered stripe phase.

\section{LOCALIZATION IN 2D SYSTEMS}

In two dimensions arbitrarily weak disorder can localize all electronic states. This famous result follows from the single parameter scaling theory of localization [8]. This theory is justified, when interactions are negligible. In the weak disorder limit $\left(k_{F} l \gg 1\right)$ the conductivity at low temperature is given by

$$
\sigma=\sigma_{0}-\frac{1}{\pi} \frac{e^{2}}{h} \ln \left(\tau_{\phi} / \tau\right),
$$

where $\sigma_{0}$ is the 'classical' Drude conductivity, and the logarithmic term is due to quantum corrections, with $\tau$ the (elastic) scattering time, and $\tau_{\phi}$ the dephasing time. These "weak localization" corrections are due to quantum interferences for electrons which are diffusing along paths containing closed loops (coherent backscattering). Dephasing is due to inelastic processes, with $1 / \tau_{\phi} \propto T^{p}$, leading to a correction to conductivity, which is logarithmic in temperature, $\delta \sigma=-\left(e^{2} / h\right)(p / \pi)|\ln (T \tau)|$.

However, single parameter scaling fails in presence of interactions, where a scaling theory including electron interactions is needed. Such a theory has been put forward by Finkel'stein [9]. In perturbation theory, new singular contributions to the conductivity are found, which are - in $2 \mathrm{~d}-$ proportional to $\log T$. These singular corrections to the conductivity are due to the interplay between disorder and interaction and arise because on distances that are larger than the mean free path electrons move slowly and have more time to interact with each other.

The correction to the resistivity due to this mechanism is

$$
\delta \rho=\frac{1}{\pi} \rho^{2}\left\{1+3\left[1-\frac{1+\gamma_{2}}{\gamma_{2}} \ln \left(1+\gamma_{2}\right)\right]\right\}|\ln (T \tau)|
$$

where " 1 " is due to interactions in the singlet channel, and " $3[\cdots]$ " are due to the triplet channels. The universal value of the singlet amplitude is due to the long range nature of the Coulomb interaction, since after screening, the dimensionless interaction equals in the long wavelength limit always one, $(\mathrm{d} n / \mathrm{d} \mu) V(q) /[1+(\mathrm{d} n / \mathrm{d} \mu) V(q)]=1 . \gamma_{2}$ is an interaction parameter which is related to the Landau parameter $-A_{a}^{0}$.

Analyzing the renormalization group flow, a metal insulator transition has been found, and a phase with finite resistance at zero temperature exists [9.:10]. The interacting system avoids localization, since the triplet amplitude becomes relevant under scaling $\left(\gamma_{2} \rightarrow \infty\right)$. The disordered Fermi liquid tends to form ferromagnetic polarons (local moments). Experimental evidence for enhanced spin fluctuations has been found near the metal-insulator 
transition in 3d Si:P. Experimental prove for large spin fluctuations near the recently discovered metal-insulator transition in 2d MOSFETs is still lacking, but the effects are currently discussed.

In the context of underdoped LSCO it is important that a magnetic field drastically modifies the above scenario. A magnetic field, besides suppressing coherent backscattering due to orbital effects, also suppresses the triplet $M= \pm 1$ due to Zeeman splitting. As a consequence, spin fluctuations remain small and $\gamma_{2} \rightarrow 1$ under renormalization. Thus the singlet term dominates leading to an insulating behavior as indeed seen in experiments in the low temperature regime [1]. We shall argue below that an analogous result can be envisaged in the extreme underdoped regime even in the absence of magnetic field.

\section{APPLICATION TO CUPRATES}

In standard studies of localization the magnetoresistance is a main probe for extracting important informations on both backscattering and interaction effects. Unfortunately, in cuprates many different contributions to the magnetoresistance have been observed related to the superconducting fluctuations [11] and to the peculiar behavior of the Hall conductance [12]. These effects may mask the localization contributions and thus make an interpretation difficult. Therefore we concentrate in the following on the temperature dependence of the resistance under conditions, where possibly the above complications are not present.

We refer to two types of experiments. Extremely underdoped LSCO $(x<0.04)$, which is non-superconducting even in absence of magnetic fields, and the system near optimum doping, but large magnetic field $(0.04<x \leq 0.16)$. In both cases $\log T$ behavior in the resistivity or conductivity has been observed, suggesting that the physics of disorder and interaction in two dimensions is relevant. However, despite a substantial anisotropy, the LSCO materials are bulk systems of weakly coupled layers. This raises the relevant issue of the effective dimensionality of LSCO with respect to localization. Weak localization in a nearly two dimensional metal has been considered by Abrikosov 13. We performed a similar calculation for the interaction contribution. We generalized the model of c-axis transport of Ref. [13] incorporating interplanar disorder as discussed in Ref. [14]. We found that the system behaves two-dimensional, when the tunneling time $\tau_{\text {tun }}$ between layers is larger than the time of the slowest processes which are contributing to localization. For weak localization, the relevant time scale is the phase coherence time $\tau_{\phi}$, whereas the time scale for the interaction contribution, which is the relevant one in high magnetic field, is $\hbar / T$. Therefore the cross over from $3 \mathrm{~d}$ to $2 \mathrm{~d}$ is defined by $1 / \tau_{\text {tun }} \sim \max \left(T / \hbar, 1 / \tau_{\phi}\right)$. The tunneling rate is hard to estimate directly, since it is not clear if processes which conserve in-plane momentum dominate, $\hbar / \tau_{\text {tun }}=2\left|t_{\perp}\right|^{2} \tau / \hbar$, or momentum non-conserving processes dominate, for which $\hbar / \tau_{\text {tun }}=2 \pi|V|^{2} N(0)$. Here $t_{\perp}$ and $V$ are tunneling amplitudes, $\tau$ the quasiparticle lifetime and $N(0)$ is the density of states. More conveniently, the tunneling rate is determined from the c-conductivity, since $\sigma_{c}=2 e^{2}\left(1 / \tau_{\text {tun }}\right) N(0) c$. Inserting the free electron value for the $2 \mathrm{~d}$ density of states with $m^{*} / m \simeq 3$ and $c=6.5 \AA$ in LSCO we find for $\rho_{c}=3 \cdot 10^{-2} \Omega \mathrm{cm}$ a tunneling rate of $\hbar / \tau_{\text {tun }} \simeq 20 \mathrm{~K}$. In Ref. [1] the c-resistivity at $20 \mathrm{~K}$ was between $\rho_{c} \simeq 2 \cdot 10^{-2} \Omega \mathrm{cm}(x=0.22)$ and $\rho_{c} \simeq 3 \Omega \mathrm{cm}(x=0.08)$. By comparing $\hbar / \tau_{\text {tun }}$ to the temperature at $20 \mathrm{~K}$, we conclude that the samples near optimal doping at $x \simeq .17$ are near the dimensional cross-over from $3 \mathrm{~d}$ to $2 \mathrm{~d}$. The underdoped samples are presumably still 
two-dimensional at $20 \mathrm{~K}$, whereas the overdoped samples may be three-dimensional. In this case a $\sqrt{T}$ correction to the conductivity at low temperature instead of $\log T$ is expected.

Preyer et al. [6] reported the conductivity in highly underdoped $\mathrm{La}_{2-\mathrm{x}} \mathrm{Sr}_{\mathrm{x}} \mathrm{CuO}_{4}$. For a sample with $x=0.04$ they found a logarithmic correction to the conductivity

$$
\sigma=\sigma_{0}-\frac{\lambda}{\pi} \frac{e^{2}}{h}\left|\ln \left(T / T_{0}\right)\right|
$$

with $\lambda \approx 0.7$ over more than a decade of temperature, from $\sigma \approx 0.5 e^{2} / h$ down to $\sigma \approx 0.1 e^{2} / h$, between $\sim 100 \mathrm{~K}$ and $\sim 10 \mathrm{~K}$, where a crossover to variable range hopping was observed. This seems to be in good agreement with standard localization theory. The magnetoresistance is negative and isotropic. Assuming that weak localization is relevant, the isotropic magnetoresistance is not consistent with conventional theory. On the other hand, conventional theory is build for a non-magnetic Fermi liquid, whereas here a theory in a doped anti-ferromagnet is needed, with peculiar quasi-particles (hole pockets). Also in absence of long range order conventional theory has to be modified. A short magnetic correlation length makes the system similar to a spin-glass. The random magnetic field in a spin-glass is assumed to suppress quantum corrections to the conductivity from coherent backscattering and from interactions in the triplet channels. Quantum corrections in the remaining interaction singlet channel are field independent, which suggests that the experimentally seen magnetoresistance is due to a different mechanism. Although a full theory of localization in a doped anti-ferromagnet is lacking, the presence of a $\log T$ of the correct magnitude, and the correct scale of the crossover to strong localization strongly suggests that the physics of disorder and interactions is here relevant.

Ando et al. [1] studied LSCO for higher doping, suppressing superconductivity by strong magnetic fields with pulses of up to $60 \mathrm{~T}$. There is practically no magnetoresistance in the normal state, i.e. once superconductivity is destroyed the resistance saturates. Below $x \approx$ 0.16 they found an insulating behavior at low temperature, i.e. $\delta \rho / \delta T<0$. Both in ab and c direction a $\log T$ in the resistance was found,

$$
\rho_{\mathrm{ab}}=\rho_{\mathrm{ab}}^{0}+\alpha\left|\ln T / T_{0}\right| \text { and } \rho_{\mathrm{c}}=\rho_{\mathrm{c}}^{0}+\beta\left|\ln T / T_{0}\right|,
$$

where $\alpha$ and $\beta$ are sample dependent. Analyzing the amplitude of the $\log T$ near the onset $\left(\approx 20 \mathrm{~K}\right.$ for $\left.\rho_{\mathrm{ab}}\right)$, i.e. calculating the "interaction constant" $\lambda_{\exp }$ according to

$$
\frac{1}{\rho_{\mathrm{ab}}} \frac{\delta \rho_{\mathrm{ab}}}{\delta|\ln T|}=\frac{\lambda_{\mathrm{exp}}}{\pi} \frac{\rho_{\mathrm{ab}}}{h / e^{2}}
$$

we found from experimental data of Ref. [1] $\lambda_{\exp } \approx 2-5$. Standard localization theory predicts in high magnetic field

$$
\lambda_{\text {theo }}=1+\left[1-\frac{1+\gamma_{2}}{\gamma_{2}} \ln \left(1+\gamma_{2}\right)\right]
$$

which is of order one, but never larger than two, since stability of the Fermi liquid requires $\gamma_{2}>-1$. Apparently $\lambda_{\exp }$ and $\lambda_{\text {theo }}$ are of the same order of magnitude.

To the first view the experiments seem to be in reasonable agreement with theory. There are, however, a number of problems: (a) While theory predicts $\log T$ in the conductivity, it 
is experimentally seen in a large range of resistivity. (b) The ratio $\rho_{\mathrm{ab}} / \rho_{\mathrm{c}}$ does not depend on temperature, i.e. the $\beta$-function in ab- and c-direction is the same, $\delta \ln \rho_{\mathrm{c}} / \delta \ln T=$ $\delta \ln \rho_{\mathrm{ab}} / \delta \ln T$. This is predicted for anisotropic, but three dimensional localization [15. In the temperature region of two dimensional localization a logarithmic correction to the c-conductivity is expected due to the corrections to the tunneling density of states, $N$. Explicitly working out the theory, we found $\delta \ln \rho_{c} / \delta \ln T=2 \delta \ln N / \delta \ln T$, which in general differs from the correction to the resistivity in ab-direction. (c) A third problem arises from a quantitative analysis of the amplitude of the $\log T$, which is of the right order of magnitude, but nevertheless is too large.

Further investigating problem (c), we found an intriguing relation between the experimentally measured amplitude of the log-corrections $\lambda_{\exp }$ and the amount of disorder as obtained from the absolute value of resistivity at some fixed temperature $\rho\left(T=T_{0}\right) \equiv \rho_{0}$. In Tab.I we report $\lambda_{\exp }$ for a number of samples, comparing "clean" (low $\rho_{0}$ ) and "dirty" samples of the same material and dopant concentration [1,7, 16]. Whereas $\lambda_{\text {theo }}$ is independent of disorder, the experimentally determined value decreases with increasing dirtiness: As shown in the table the product $\rho_{0} \lambda_{\exp }$ is nearly disorder independent. Moreover, $\rho_{0} \lambda_{\exp }$ seems to decrease with increasing doping. For the four LSCO samples we report in Tab.I the product $\rho_{0} \lambda_{\exp } x^{2}$ is roughly independent from disorder and doping.

A second observation is, that various features of the anomalous localization can be described phenomenologically by using the Drude formula for the conductivity and taking the scattering rate from the ansatz

$$
\frac{1}{\tau}=\frac{1}{\tau_{0}}\left(1+a\left|\ln T / T_{0}\right|\right)
$$

This logarithmically enhanced scattering rate appears directly in the resistivity, not in the conductivity, and is therefore consistent with the property outlined in point (a) above. Moreover the constant ratio of $\rho_{\mathrm{ab}} / \rho_{\mathrm{c}}$ can be reconciled with a $\log T$ correction which is typical of two dimensional systems (problem (b) outlined above) by the assumption (7) if tunneling between planes is dominated by momentum conserving processes. Finally, if dirtiness only affects $1 / \tau_{0}$, but not the logarithmic term, the amplitude of the $\log T$ as a function of disorder behaves according to the experimental observation discussed above, $\rho_{0} \lambda_{\exp }=$ const.

\section{DISCUSSION}

We discussed some features of transport experiments in LSCO compounds in the normal state. On the one hand the $\log T$ correction in strongly underdoped LSCO $(x \approx 0.04)$ appears to be consistent with standard localization theory, although an explanation for the magnetoresistance is still lacking. On the other hand, for higher doping, $0.04 \lesssim x \lesssim 0.16$, standard theory is not able to explain the experiments.

There are several reasons why the conventional "old fashioned" localization theory is not expected to work well in strongly correlated anisotropic systems like the cuprates. One first possibility is that the cuprates can not be described by the Fermi liquid theory as already pointed out in the introduction [17]. If this is the case, a new localization theory starting from a clean non-Fermi liquid system should be devised [2,5]. Alternatively, a 
singular interaction could be responsible for both the disruption of the Fermi liquid and of the anomalous localization effects. Mirlin and Wölfle [18] reported anomalous localization effects within a gauge field theory [19], where particles interact via a singular transverse gauge field propagator $\propto 1 /\left(-\mathrm{i} \omega \sigma+\chi q^{2}\right)$. At low temperature a $\log T$ correction has been found, with an amplitude that depends on resistance itself, $\lambda \propto \ln (1 / \rho)$.

In the quantum critical point (QCP) scenario of high $T_{c}$ superconductivity, a QCP exists near optimum doping, with an "ordered" stripe phase in the underdoped regime [20 22]. In this context, possible sources of singular interactions are soft modes from dynamical stripes, or critical fluctuations near the QCP. Specifically, the interaction near the stripe critical wave-vector $\mathbf{q}_{c}$ may be parameterized as [20]

$$
\Gamma(\mathbf{q}, \omega) \approx-\frac{A}{\omega_{\mathbf{q}}-\mathrm{i} \omega},
$$

where $\omega_{\mathbf{q}} \approx B\left(\left|\mathbf{q}-\mathbf{q}_{c}\right|^{2}+\kappa^{2}\right)$. Quantum corrections to the conductivity due to exchange of these fluctuations are, to first order in $\Gamma(\mathbf{q}, \omega)$, proportional to the Fermi-surface average of the static interaction, $\Gamma_{\mathrm{QCP}}=\langle\Gamma(\mathbf{q})\rangle_{F S} \propto-A \log \left(k_{F} / \kappa\right)$. The interaction is attractive, leading to an increase of localization. For large $\Gamma_{\mathrm{QCP}}$ quantum corrections have to be calculated beyond first order. However standard theory [9] does not apply here, due to the strong frequency dependence of the interaction. We have preliminary results indicating, that quantum $\left(\omega_{\mathbf{q}}>T\right)$ and classical $\left(\omega_{\mathbf{q}}<T\right)$ fluctuations contribute differently to localization.

Finally one should also think at the possibility of a non-conventional source of disorder. In particular, if a disordered nearly static stripe phase is realized in these systems one should, besides the conventional impurity disorder, also consider the disorder coming from domain boundaries and other topological defects of the striped textures.

An appealing possibility is that a disordered stripe phase could be responsible for the anomalous localization behavior both by introducing a singular scattering between the electrons and by providing topological disorder via domain boundaries. As a consequence, any mechanism like impurity or lattice pinning of (static or slow) stripe fluctuations is expected to reduce the amplitude of the effective interaction and the $\log T$ corrections, in agreement with the central observation of the present work that $\rho_{0} \lambda_{\exp }$ is nearly constant. This expectation is supported by the observation, that at $x=1 / 8$, where stripes are more ordered, the $\log T$ is less strong and the resistance saturates at low temperature [1.23], as in $\mathrm{La}_{1.48} \mathrm{Nd}_{0.4} \mathrm{Sr}_{0.12} \mathrm{CuO}_{4}$ [24].

The very speculative character of these considerations calls for a detailed theory, which is not available at the moment. 


\section{REFERENCES}

[1] Y. Ando, G.S. Boebinger, A. Passner, T. Kimura and K. Kishio, Phys. Rev. Lett. 75, 4662 (1995); G.S. Boebinger et al., Phys. Rev. Lett. 77, 5417 (1996).

[2] P.W. Anderson, T.V. Ramakrishnan, S. Strong, and D.G. Clarke, Phys. Rev. Lett. 77, 4241 (1996).

[3] A.S. Alexandrov, preprint, cond-mat/9610065.

[4] B.K. Chakraverty, J. Ranninger, and D. Feinberg, Phys. Rev. Lett. 81, 433 (1998); A.S. Alexandrov, preprint, cond-mat/9807185 (comment).

[5] C.M. Varma, Phys. Rev. Lett. 79, 1535 (1997).

[6] N.W. Preyer, M.A. Kastner, C.Y. Chen, R.J. Birgenau, and Y. Hikada Phys. Rev. B 44, 407 (1991); B. Keimer et al, Phys. Rev. B 46, 14034 (1992).

[7] Y. Ando et al., Phys. Rev. B 56, 8530 (1997).

[8] E. Abrahams, P.W. Anderson, D.C. Licciardello, and T.V. Ramakrishnan Phys. Rev. Lett. 42, 673 (1979).

[9] A.M. Finkel'stein JETP 57, 97 (1983); C. Castellani, C. Di Castro, P.A. Lee, and M. Ma, Phys. Rev. B 30, 527 (1984). For a review see: P.A. Lee and T.V. Ramakrishnan, Rev. Mod. Phys. 57, 787 (1985); D. Belitz and T.R. Kirkpatrick, Rev. Mod. Phys. 66, 261 (1994).

[10] C. Castellani, C. Di Castro, and P.A. Lee, Phys. Rev. B 57, 9381 (1998).

[11] A. Varlamov et al., Adv. in Phys., in press (1998)

[12] A.W. Tyler et al., Phys. Rev. B 57, 728 (1998).

[13] A.A. Abrikosov, Phys. Rev. B 501415 (1994).

[14] A.G. Rojo and K. Levin, Phys. Rev. B 4816861 (1993).

[15] P. Wölfle and R.N. Bhatt, Phys. Rev. B 30, 3542 (1984); R.N. Bhatt, P. Wölfle, and T.V. Ramakrishnan, Phys. Rev. B 32, 569 (1985).

[16] T.W. Jing, N.P. Ong, T.V. Ramakrishnan, J.M. Tarascon, and K. Remschnig, Phys. Rev. Lett. 67, 761 (1991).

[17] P.W. Anderson, Science 235, 1196 (1987); Phys. Rev. Lett. 64, 1839 (1990); 65, 2306 (1990).

[18] A. Mirlin and P. Wölfle, Phys. Rev. B 55, 5141 (1997).

[19] N. Nagaosa and P.A. Lee, Phys. Rev. Lett. 64, 2450 (1990); P. A. Lee and N. Nagaosa, Phys. Rev. B 46, 5621 (1992);

[20] C. Castellani, C. Di Castro, and M. Grilli, Phys. Rev. Lett. 75, 4650 (1995); A. Perali, C. Castellani, C. Di Castro, and M. Grilli, Phys. Rev. B 54, 16216 (1996).

[21] For experimental evidence of a stripe phase in the cuprates see the contributions to this conference by A. Bianconi and by J.M. Tranquada. For theoretical aspects see instead the contributions by C. Di Castro, V.J. Emery, and J. Zaanen.

[22] Different QCPs have been suggested by C.M. Varma, Physica C 26339 (1996); P. Montoux and D. Pines, Phys. Rev. B 50, 16015 (1994) and references therein.

[23] C. Castellani, C. Di Castro and M. Grilli, cond-mat/9709278.

[24] J.M. Tranquada, J.D. Axe, N. Ichikawa, Y. Nakamura, S. Uchida, and B. Nachumi, Phys. Rev. B 54, 7489 (1996). 


\section{TABLES}

\begin{tabular}{|c|c|c|c|}
\hline \hline$\rho_{0}=.26$ & $\lambda_{\exp }=5.2$ & LSCO,$x=0.08$ & $\rho_{0} \lambda_{\exp }=1.3$ \\
$\rho_{0}=.83$ & $\lambda_{\exp }=1.87$ & LSCO,$x=0.08$ & $\rho_{0} \lambda_{\exp }=1.5$ \\
\hline$\rho_{0}=.15$ & $\lambda_{\exp }=4.2$ & LSCO,$x=0.13$ & $\rho_{0} \lambda_{\exp }=0.63$ \\
$\rho_{0}=.20$ & $\lambda_{\exp }=3.1$ & LSCO,$x=0.13$ & $\rho_{0} \lambda_{\exp }=0.60$ \\
\hline$\rho_{0}=.046$ & $\lambda_{\exp }=2.5$ & Bi2201, $x=?$ & $\rho_{0} \lambda_{\exp }=0.115$ \\
$\rho_{0}=0.161$ & $\lambda_{\exp }=0.6$ & Bi2201, $x=?$ & $\rho_{0} \lambda_{\exp }=0.10$ \\
\hline \hline
\end{tabular}

TABLE I. Interaction "constant" $\lambda_{\exp }$ as determined from clean and dirty LSCO [1,7] and Bi2201 [7, 16], see Eq. (5). The dopant concentration of the Bi compounds, i.e. the concentration of holes in the $\mathrm{CuO}$ planes, is not clear to us, however the samples are assumed to be overdoped. $\rho_{0}$ is the sheet resistance in units of $h / e^{2}$ at $T \approx 20 \mathrm{~K}$. Note that the product $\rho_{0} \lambda_{\exp }$ assumes the same value for clean and dirty samples. 